\title{
ARTIGO
}

\section{CONSIDERAÇÕES SOBRE O USO DE CONCEITOS DA MECÂNICA DA FRATURA PARA O PROJETO DE PAVIMENTOS DE CONCRETO DE CIMENTO}

\section{Jorge Barbosa Soares}

Universidade Federal do Ceará - UFC

\section{RESUMO}

A resistência à tração usada no dimensionamento de estruturas de concreto, como pavimentos, tem sido tradicionalmente determinada a partir de corpos de prova de laboratório. É sabido que valores de resistência obtidos a partir de corpos de prova geometricamente diferentes podem ser bastante diferentes. Naturalmente, pode-se questionar a aplicação da resistência obtida pelos corpos de prova convencionais a uma estrutura real. Esta deficiência está relacionada aos efeitos de tamanho e geometria da peça na resistência do material. Dado que parâmetros de fratura podem ser usados na determinação da resistência à tração do concreto, é apresentado um método simplificado para a determinação destes parâmetros, baseado na teoria da mecânica da fratura. A determinação da resistência à tração por parâmetros de fratura é também discutida conforme se aplica a requerimentos de profundidade de corte em pavimentos de concreto com juntas.

\section{ABSTRACT}

The tensile strength used in the design of concrete structures, such as concrete pavement systems, has typically been determined based on small test specimens. It is well known that strength values obtained from geometrically different specimens can be largely different. Naturally, one must question the applicability of strength values obtained from these conventional specimens to an actual structure. 
This deficiency is encompassed within effects of specimen (or structure) size and geometry on the strength. Given that fracture parameters can be used to determine concrete strength, a simplified tension test method, based on the fracture mechanics theory, is presented to determine fracture parameters. Applications of the method to determine concrete fracture resistance are also discussed as they apply to concrete pavement sawcut depth requirements.

\section{INTRODUÇÃO}

A falha de materiais quasi-frágeis é caracterizada por uma grande não linearidade. Em contraste, materiais perfeitamente frágeis possuem uma curva tensão-deformação elástica linear até o ponto de máxima tensão quando é observado o colapso (falha abrupta). Vários estudos têm sido realizados nas últimas duas décadas para entender o mecanismo de falha em materiais quasi-frágeis. Estes incluem uma gama de materiais como concretos, cerâmicas, rochas, gelo, compostos e alguns metais. $\mathrm{O}$ presente artigo trata principalmente de materiais de concreto de cimento portland, com aplicação a pavimentos, apesar dos desenvolvimentos discutidos também serem aplicáveis a outros materiais quasi-frágeis. Um dos objetivos do presente artigo é contribuir para uma discussão mais aprofundada na comunidade de pavimentação no Brasil sobre a aplicação da teoria da mecânica da fratura a pavimentos de concreto. A discussão deve estender-se a pavimentos asfálticos, ressaltando-se que já há referências nacionais sobre aplicações da referida teoria a estes pavimentos. A aplicação prática dos desenvolvimentos da teoria da mecânica da fratura ainda é insipiente mesmo nos países aonde estes desenvolvimentos vêm sendo realizados.

O rompimento de uma peça de concreto envolve a formação de trinca(s) e a propagação estável desta(s) até uma carga limite ser atingida no caso de carregamento monotônico. A fadiga no concreto envolve um dano progressivo e irreversível associado a propagação estável $\mathrm{da}(\mathrm{s})$ trinca(s) sob um carregamento repetido. Ambas as falhas, monotônica e por fadiga, têm sido estudadas sob a luz da teoria da mecânica da fratura. Entre as razões para a utilização desta teoria no estudo da falha em concreto estão: (1) o chamado efeito de tamanho que indica que a resistência é diferente para peças de 
tamanhos diferentes; (2) o fato das resistências medidas no laboratório em corpos de prova geometricamente diferentes serem consideravelmente diferentes e, também, (3) da medida de resistência obtida em laboratório não ser representativa da resistência na configuração da estrutura real em campo devido aos efeitos de tamanho e geometria, e finalmente, (4) o fato da resistência não servir como um indicador da fragilidade do material.

Diferentemente dos materiais frágeis (vidro, por exemplo), que rompem por colapso uma vez que uma trinca é formada, materiais quasi-frágeis como o concreto podem sustentar uma propagação estável de trincas antes de ocorrer a falha por colapso. Parâmetros de fratura podem ser usados na caracterização da fragilidade do material. Estes parâmetros podem também ser usados na determinação da resistência à tração de uma peça em qualquer configuração. Ainda assim, o projeto de estruturas de concreto nas quais a resistência à tração é um fator importante, como barragens e pavimentos, não é atualmente baseado na análise pela teoria da mecânica da fratura. Uma razão é a complexidade existente na determinação dos parâmetros de fratura em laboratório. É discutido neste artigo um ensaio simplificado, com potencial para utilização na prática da engenharia, para a determinação dos referidos parâmetros. É ainda apresentado o uso dos parâmetros de fratura na determinação da resistência à tração e como critério de dimensionamento para especificações da profundidade de corte em pavimentos de concreto com juntas.

\section{REVISÃO BIBLIOGRÁFICA}

Tem sido prática comum no controle de qualidade de pavimentos de concreto em rodovias e aeroportos considerar a resistência à tração obtida a partir de pequenos corpos de prova (ensaios a flexão em particular) como representativa da resistência do concreto no pavimento real em campo. Esta prática tem sido justificada considerando que o sistema do pavimento é carregado à flexão semelhantemente a um corpo de prova prismático. Tensões e deformações numa placa de concreto podem ser determinadas pelas soluções fechadas de Westergaard (Westergaard, 1926; Ioannides et al., 1985), gráficos de influência de Pickett e Ray (1951) e programas 
de elementos finitos (Huang, 1974). As soluções de Westergaard e os gráficos de Pickett e Ray para cálculo das tensões máximas consideram uma placa infinitamente larga sobre uma fundação Winkler (líquido denso). Independentemente da representação escolhida para a fundação, as condições de carregamento e contorno em um pavimento de concreto e nos corpos de prova convencionais são bem diferentes. Estas diferenças fazem com que a consideração da resistência do concreto na estrutura real com base na resistência dos corpos de prova de laboratório seja questionável. Mesmo assumindo condições idênticas de carregamento e suporte para as configurações de laboratório e de campo, o efeito do tamanho da peça na resistência precisaria ser considerado.

No laboratório, os valores de resistência obtidos em corpos de prova geometricamente iguais variam com o tamanho destes. Ainda, valores de resistência obtidos em corpos de prova geometricamente diferentes, mesmo com dimensões características iguais (diâmetro de um cilindro = altura de um corpo de prova prismático), também são diferentes (Mindess e Young, 1981). Portanto, o parâmetro resistência determinado em qualquer corpo de prova é tendencioso devido (1) ao efeito de geometria, significando que o concreto ensaiado em um corpo de prova cilíndrico à tração indireta fornecerá um valor de resistência diferente do mesmo concreto ensaiado em outro tipo de corpo de prova como o prismático; e (2) ao efeito de tamanho, o que significa que a resistência determinada a partir dos pequenos corpos de prova não pode ser diretamente aplicada como a resistência do material existente em uma estrutura maior (Tang et al., 1992). Qualquer teoria que usa a tensão limite (resistência) como o critério de falha assume que corpos de diferentes geometrias e tamanhos falham quando a tensão nominal atinge a tensão limite. Esta hipótese é contrariada pelas observações experimentais dos efeitos de geometria e tamanho no valor da resistência (Wright e Garwood, 1952; Mindess e Young, 1981).

A resistência nominal de uma peça é definida como $\sigma_{N}=c_{n} P /[b d]$, onde $P$ é a carga de ruptura, $b$ é a largura da peça e $d$ é a dimensão característica (espessura numa placa, altura num corpo de prova prismático, diâmetro num cilindro). A constante $c_{n}$ é usualmente definida de modo a tornar $\sigma_{N}$ igual a tensão de ruptura (resistência) 
numa estrutura ou corpo de prova. Para o ensaio em corpo de prova prismático de um cutelo, a resistência nominal é definida escolhendo-se $c_{n}=1,5 \mathrm{l} / \mathrm{d}$ ( $l$ sendo a distância entre os cutelos de suporte) de forma que $\sigma_{N}=(1,5 l / d) P /[b d]$. No caso de carregamento nos terços do vão com ruptura no terço médio da distância entre os elementos de apoio, $\left.\sigma_{N}=P l \Lambda b d^{2}\right]$ e, portanto, $c_{n}=l / d$.

Dado que o critério de resistência não considera os efeitos de tamanho e geometria, critérios alternativos devem ser investigados, sendo àqueles baseados na mecânica da fratura uma opção já consolidada no estudo de outros materiais. Vários autores têm aplicado a mecânica da fratura ao concreto para diferenciar os efeitos de tamanho e geometria na determinação da resistência à tração (Hillerborg et al., 1976; Bazant, 1984; Jenq e Shah, 1985; Nallathambi e Karihaloo, 1986; Swartz e Refai, 1988; Tang et al., 1996; Soares e Zollinger, 1997a, 1997b). Os modelos de fratura desenvolvidos para concreto foram divididos por Planas e Elices (1990) em dois grupos: modelos elástico lineares modificados e modelos de amolecimento progressivo (progressive softening models). No primeiro grupo estão a lei do efeito de tamanho (size effect law) (Bazant, 1984; Bazant e Kazemi, 1990a), e o modelo de dois parâmetros (two-parameter fracture model) de Jenq e Shah (1985). O segundo grupo inclui modelos de banda da trinca (Bazant e Oh, 1983) e modelos de zona coesiva (Hillerborg et al., 1976). Relações entre os diferentes modelos são encontradas na literatura (Elices e Planas, 1989; Planas e Elices, 1989; Shah et al., 1995). Aplicações específicas a pavimentos são encontradas em Lin e Folias (1975), Ramsamooj (1993), Zollinger et al. (1994), Soares (1997) e Ioannides (1997). No Brasil, Rodrigues (1991) e Pinto (1991) estudaram a aplicação da teoria da mecânica da fratura a pavimentos asfálticos.

Baseado no modelo de fratura de dois parâmetros e na lei do efeito de tamanho, o Texas Transportation Institute (TTI) desenvolveu e vem aplicando ensaios para a determinação dos parâmetros de fratura (Tang et al., 1996; Soares, 1997). Os resultados indicam um potencial para o melhoramento da caracterização do concreto utilizando estes parâmetros que podem ainda ser usados para determinar a resistência considerando os efeitos de tamanho e geometria. Portanto, o uso de qualquer tipo de correlação empírica 
entre a resistência do concreto na estrutura real e a resistência obtida em laboratório é desnecessário. Isto é particularmente importante para a área de pavimentação onde tem havido um interesse crescente de se usar a resistência à compressão para controle de qualidade na forma de correlações com a resistência à flexo-tração. Apesar das resistências à compressão ou flexo-tração em corpos de prova poderem ser usadas para controle de qualidade, do ponto de vista do dimensionamento, é importante notar que estas quantidades não representam a resistência como é configurada na estrutura do pavimento real.

De acordo com a Mecânica da Fratura Linearmente Elástica (MFLE), a fratura ocorre a partir de uma trinca pré-existente quando as tensões na ponta da trinca atingem valores críticos. Estas tensões são associadas a um parâmetro chamado fator de intensidade de tensões $(K)$ que pode ser compreendido como uma força guia da trinca (crack driving force). Ele é proporcional ao carregamento e depende do comprimento inicial da trinca e da geometria e tamanho da estrutura. São bastante abordados na literatura o desenvolvimento $\mathrm{e}$ as aplicações do parâmetro $K$, bem como a sua associação a energia elástica liberada pelo corpo devido a propagação da trinca (Tada et al., 1985; Broek, 1986; Shah et al., 1995).

No modo I (abertura das faces da trinca), o crescimento instável da trinca ocorre quando o fator de intensidade de tensões $K_{I}$ atinge um valor crítico chamado de tenacidade à fratura $K_{l c}$. Este último expressa a capacidade de um material perfeitamente frágil, na presença de uma trinca inicial, de resistir a propagação desta trinca. A propagação não ocorre enquanto $K_{I}<K_{I c}$. Portanto, $K_{I}$ está para tensão solicitante assim como $K_{I c}$ está para resistência do material, com a vantagem porém de levar em consideração a geometria e o tamanho da peça. $\mathrm{Na}$ abordagem elástica linear, válida para materiais perfeitamente frágeis, a resistência nominal $\left(\sigma_{N}\right)$ pode ser calculada por:

$$
\sigma_{N}=\frac{K_{I c}}{\sqrt{\pi a_{0}} F\left(\alpha_{0}\right)}
$$


em que: $a_{0}=$ comprimento inicial da trinca; $\alpha_{0}=$ razão $a_{0} / d$ ( $d$ sendo a dimensão característica) e $F(\alpha)=$ fator de geometria. Este fator, função das condições de carregamento e contorno da peça em questão, está disponível em manuais para geometrias simples (Tada et al., 1985) ou pode ser determinado pelo método dos elementos finitos (método da propagação virtual da trinca, elementos singulares, etc.) (Chan et al., 1970; Parks, 1974).

Para peças geometricamente similares, $\alpha_{0}$ e $F\left(\alpha_{0}\right)$ são constantes. Tomando-se o logarítmico na equação (1) tem-se que o $\log \left(\sigma_{N}\right)$ é uma função linear do logarítmico do tamanho da peça $d$ (espessura de uma placa, por exemplo), conforme mostrado na reta com coeficiente angular $-1 / 2$ na Figura 1. A possibilidade de se mostrar o efeito do tamanho da peça na resistência é a diferença fundamental entre a MFLE e a tradicional teoria da ruptura que assume a resistência como uma constante com respeito ao tamanho da peça conforme indicado pela linha horizontal na Figura 1.

Porém, o efeito do tamanho na resistência em materiais quasi-frágeis como o concreto não segue uma linha reta como mostrado na Figura 1. Ele segue uma curva de transição que se aproxima do critério da ruptura para pequenos corpos de prova e se aproxima do limite definido pela MFLE para grandes estruturas. Esta curva de transição engloba as estruturas do tamanho que interessam na maioria das aplicações práticas (Bazant e Kazemi, 1990a, 1990b).

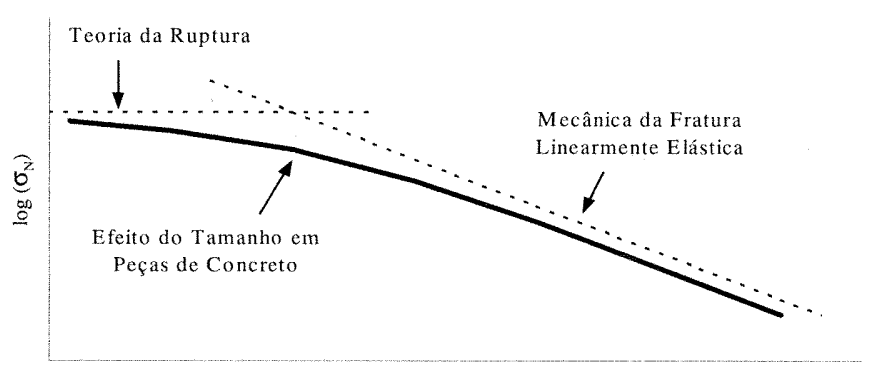

$\log (d)$

Figura 1: Efeito do tamanho em peças de geometrias similares e tamanhos diferentes 
Diferentemente da MFLE, o efeito do tamanho da peça na resistência do concreto é devido a presença de uma região aonde se processa a fratura na ponta da trinca (zona de processamento da fratura). $\mathrm{O}$ elevado campo de tensões nesta zona gera microfissuras. A propagação destas microfissuras é protegida pela existência de poros preenchidos com água, vazios e trincas de retração que consomem parte da energia externa proveniente do carregamento. Tal fenômeno (microcrack shielding) proporciona maior tenacidade ao concreto. Um crescimento instável da trinca não ocorre até a zona de processamento da fratura desenvolver um comprimento crítico.

Vários modelos modificam a MFLE introduzindo um comprimento de trinca efetivo $\left(a_{c}\right)$ formado pelo comprimento original da trinca $\left(a_{0}\right)$ mais o comprimento da zona de processamento (c), ou seja, $a_{c}=a_{o}+c$. Entres estes modelos está a lei do efeito de tamanho generalizada (Bazant e Kazemi 1990a), que propõe que a MFLE se aplica a uma estrutura de concreto infinitamente grande $(d \rightarrow \infty)$. Tal peça rompe quando $K_{I}$ atinge um valor crítico $K_{l f}$ e a zona de processamento atinge um comprimento crítico $c_{f}$, simultaneamente. $O$ parâmetro $c_{f}$ é referido como o comprimento crítico da zona de processamento para uma peça infinitamente grande.

Para materiais com zonas de processamento não desprezíveis como o concreto, $K_{I c}$ para peças finitas é reconhecidamente dependente do tamanho e pode ser calculado em termos de $K_{I f}$, ou seja, $\lim K_{l c}(\mathrm{~d} \rightarrow \infty)$ $=K_{\text {If }}$ (Bazant e Kazemi 1990a). Uma discussão mais aprofundada sobre a relação entre $K_{I c}$ e $K_{\text {If }}$ pode ser encontrada em Bazant e Kazemi (1990a, 1990b). Conforme indicado por Zollinger et al. (1993), apesar dos parâmetros de fratura $K_{\text {If }}$ e $c_{f}$ se referirem a uma peça infinitamente grande, eles podem ser aplicados de forma conservadora para dimensões de placas de concreto.

Considerando uma função de geometria adimensional $g(\alpha)=\pi \alpha c_{n}^{2}$ $F^{2}(\alpha)$, onde $F(\alpha)$ é o fator de geometria previamente mencionado e, baseado em $K_{\text {If }}$ e $c_{f}$, para qualquer tamanho finito de peça $d$, a resistência nominal pode ser determinada a partir da forma final da lei do feito de tamanho de Bazant e Kazemi (1990a): 


$$
\sigma_{N}=c_{n} \frac{K_{I f}}{\sqrt{g^{\prime}\left(\alpha_{o}\right) c_{f}+g\left(\alpha_{o}\right) d}}
$$

em que: $\sigma_{N}=c_{n} P /[b d], \alpha_{0}$ é a razão inicial $\left(a_{0} / d\right), g(\alpha)$ e sua derivada $g^{\prime}(\alpha)$ são funções da geometria da peça avaliadas para $\alpha=\alpha_{0}$. Note que uma vez que a função $g(\alpha)$ é definida como $g(\alpha)=\pi \alpha c_{n}^{2} F^{2}(\alpha)$, e portanto, $g^{\prime}(\alpha)=\pi c_{n}^{2} F^{2}(\alpha)+\pi \alpha c_{n}^{2} 2 F(\alpha) F^{\prime}(\alpha)$, o termo $c_{n}^{2}$ é fatorado para fora da raiz quadrada na equação (2) e cancelado com o $c_{n}$ no numerador. Portanto, diz-se que a seleção de $c_{n}$ não afeta a forma da lei generalizada do efeito de tamanho. Para uma discussão mais detalhada sobre a derivação de fatores de geometria bem como de outras derivações da lei do efeito de tamanho ver Soares (1997). A importância da equação (2) está no fato de, a partir dos parâmetros de fratura $\left(K_{\text {If }}\right.$ e $\left.c_{f}\right)$, poder-se determinar a resistência de uma peça levando em consideração a sua geometria, $g(\alpha)$ e $g^{\prime}(\alpha)$, e o seu tamanho, $d$.

Para identificar quantitativamente o espectro dos tamanhos nos quais os modelos não lineares de fratura devem ser aplicados, Bazant e Pfeiffer (1987) introduziram o conceito do número de fragilidade definido como $\beta=\left[g\left(\alpha_{0}\right) d\right] /\left[g^{\prime}\left(\alpha_{0}\right) c_{f}\right]$. Quando $\beta<1$, o comportamento do corpo tende para aquele assumido na análise pela ruptura, enquanto para $\beta>1$ ele tende a MFLE. Bazant e Pfeiffer (1987) sugerem que se use modelos não lineares para $0,1<\beta<10$.

\section{ENSAIOS PARA DETERMINAÇÃO DOS PARÂMETROS DE FRATURA}

Os parâmetros de fratura $K_{l f}$ e $c_{f}$ podem ser determinados experimentalmente com base na equação (2). O método do efeito de tamanho recomendado pelo RILEM (1990) tem sido usado para se determinar os parâmetros de fratura através do ensaio de corpos de prova prismáticos com um cutelo. Os corpos de prova são geometricamente similares (dimensões incluindo o comprimento da trinca inicial proporcionais), porém de tamanhos diferentes. Portanto, $\alpha_{0}, g\left(\alpha_{0}\right)$ e $g^{\prime}\left(\alpha_{0}\right)$ são constantes, mas a altura da viga, $d$, é diferente para cada corpo de prova. No procedimento do RILEM, é sugerido que a dimensão do menor corpo de prova seja maior que 3 
vezes o tamanho máximo do agregado. É ainda recomendado que a razão entre a dimensão do maior corpo de prova $d_{2}$ e do menor corpo de prova $d_{1}, d_{2} / d_{1}$, seja maior que 4 , devido a dispersão de dados tipicamente encontrada (Bazant e Kazemi, 1990a). De acordo com estas especificações, o valor da altura $d_{2}$ pode ser tão elevado de modo a ultrapassar a capacidade de prensas tipicamente encontradas em laboratórios.

Para desenvolver-se um procedimento mais prático que o método do RILEM, o método do tamanho único foi desenvolvido e vem sendo testado no TTI (Tang et al., 1996; Soares, 1997). São usados vários corpos de prova do mesmo tipo e tamanho, aonde entalhes de diferentes comprimentos são formados para simular a existência de uma trinca inicial. Todos os corpos de prova têm dimensão $d$ (altura da viga ou diâmetro do cilindro). Neste método, a equação (2) foi convertida para uma regressão linear $Y=A X+C$, em que:

$$
Y=\frac{c_{n}^{2}}{g^{\prime}\left(\alpha_{o}\right) \sigma_{N}^{2}} ; \quad X=d \frac{g\left(\alpha_{o}\right)}{g^{\prime}\left(\alpha_{o}\right)} ; \quad K_{\text {If }}=\frac{1}{\sqrt{A}} ; \quad c_{f}=\frac{C}{A}
$$

Quando corpos de prova do mesmo tamanho são usados, o requerimento colocado em $d_{2}$ e $d_{1}$ no método RILEM é traduzido para um requerimento na razão entre $X_{2}$ e $X_{1}$. Os subscritos são referentes aos corpos de prova com maior e menor valor de $X$ na equação (3), respectivamente. É apropriado para a razão $X_{2} / X_{1}$ ser maior ou igual a 4 devido a dispersão de dados tipicamente encontrada em ensaios em concreto. Uma vez que $d$ é constante, $X_{2} / X_{1}$ depende apenas da razão $g\left(\alpha_{0}\right) / g^{\prime}\left(\alpha_{0}\right)$ para os corpos de prova 1 e 2 . Razões maiores $X_{2} / X_{1}$ ou dispersões menores dos dados conduzem a linhas de regressão mais confiáveis (Soares, 1997).

O método do tamanho único desenvolvido no TTI faz uso de corpos de prova cilíndricos. Estes têm a vantagem de serem facilmente moldados ou extraídos de estruturas de concreto. $\mathrm{O}$ ensaio de tração indireta é padronizado pela ABNT (1994) e pela ASTM (1995) para obtenção da resistência à tração do concreto. Os corpos de prova cilíndricos modificados, ilustrados na Figura 2, foram desenvolvidos para a determinação dos parâmetros de fratura. A configuração 
destes corpos de prova proporciona uma razão $X_{2} / X_{1}$ suficientemente grande para a regressão linear da equação (3). O orifício aberto no corpo de prova na Figura $2 \mathrm{c}$ altera bastante a geometria do corpo de prova modificando a razão $g\left(\alpha_{0}\right) / g^{\prime}\left(\alpha_{0}\right)$ (Tang et al., 1996). Os orifícios ilustrados podem ser feitos com serra ou moldados com hastes colocadas durante a moldagem (Soares, 1997). O comprimento da trinca inicial é $2 a$ e, portanto, $\alpha_{0}=2 a / d=a / R$, aonde $d$ é o diâmetro e $R$ o raio do cilindro. Na Figura 2, $t / R$ é 0,16 , e $r / R$ para o cilindro com orifício é 0,125 ( $t$ é a largura do carregamento distribuído e $r$ o raio do orifício na Figura $2 c$ ). Os fatores de geometria, $F(\alpha)$, dos cilindros com orifício e sem orifício são dados por (Tang et al., 1996):

Com orifício : $F(\alpha)=0,964-0,026 \alpha+1,472 \alpha^{2}-0,250 \alpha^{3}$

Sem orifício : $F(\alpha)=2,849-10,451 \alpha+22,938 \alpha^{2}-14,940 \alpha^{3}$

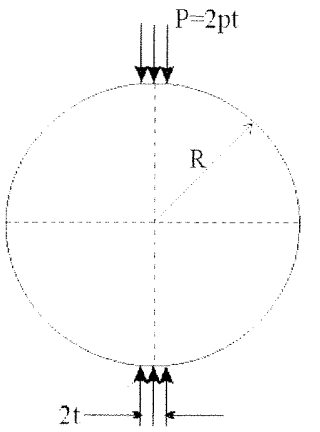

(a)

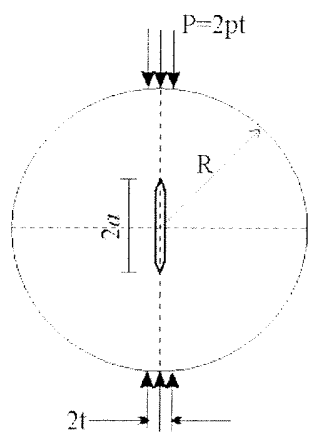

(b)

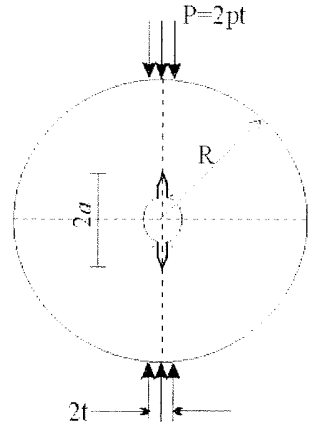

(c)

Figura 2: Corpos de prova cilíndricos

\section{RESULTADOS EXPERIMENTAIS}

O procedimento desenvolvido para a determinação dos parâmetros de fratura consiste em usar-se os corpos de prova cilíndricos ilustrados nas Figuras 2a, 2b e 2c (tipos 1, 2 e 3, respectivamente). $\mathrm{O}$ primeiro não contém trinca inicial $\left(2 a_{0}=0\right)$; o segundo contém uma trinca de $2 a_{0}=25,4 \mathrm{~mm}$ e o terceiro contem um orifício de $19,1 \mathrm{~mm}$ de 
diâmetro com entalhes de $8,8 \mathrm{~mm}$ estendendidos em cada lado do orifício, de forma que $2 a_{0}=36,7 \mathrm{~mm}$.

O entalhe no corpo de prova tipo 2 para simular a trinca inicial pode ser obtido de duas maneiras distintas: (1) uma haste de alumínio de 25,4 mm, lubrificada, é usada na moldagem do corpo de prova e extraída 24 horas após a moldagem; e (2) um rasgo de $25,4 \mathrm{~mm}$ de comprimento é feito com uma serra recíproca colocada através de um orifício de $12,5 \mathrm{~mm}$ de diâmetro aberto no centro do cilindro. Este orifício é suficientemente pequeno para não influir no campo de tensões ao redor da trinca (Tang et al., 1996). O entalhe no corpo de prova tipo 3 também pode ser obtido com uma serra recíproca colocada através de um orifício perfurado no corpo de prova. $O$ orifício com entalhes também pode ser formado por uma haste préfabricada no formato da Figura 2c. Como no caso do corpo de prova tipo 2, a haste é extraída. Soares (1997) não encontrou diferença estatisticamente significativa entre os resultados da resistência nominal quando as trincas iniciais são produzidas com hastes ou entalhes por serra, indicando que ambas as técnicas podem ser usadas. A largura de todos os entalhes é de aproximadamente $3 \mathrm{~mm}$, e o comprimento inicial do entalhe é tomado como a média do comprimento de 3 locais diferentes medidos após o ensaio.

A seguir são apresentados resultados de parte do trabalho de Soares (1997). Em uma batelada, foram moldados 7 cilindros. Cada cilindro de $30 \mathrm{~cm}$ de altura foi cortado em 3 corpos de prova de aproximadamente $10 \mathrm{~cm}$ de altura. Menores corpos de prova possibilitam um maior número de dados e tem a vantagem de serem facilmente manuseados, além de permitirem o uso de prensas de menor capacidade. Foi considerado o fato das partes superior, central e inferior do cilindro apresentarem características diferentes devido à moldagem e a cura inicial conforme indicado por Ojdrovic e Petroski (1987). Apesar do agrupamento dos dados de acordo com a posição no cilindro diminuir a dispersão dos dados, foi verificado que tratar os dados conjuntamente sem distinção da posição fornece valores dos parâmetros de fratura representativos para o material $e$ compativeis com os resultados obtidos separando os cilindros de acordo com a localização. 
Os corpos de prova foram testados à tração indireta com os entalhes perfeitamente alinhados com o eixo vertical do carregamento de modo a se obter o modo I de falha. Para o corpo de prova tipo $1\left(\alpha_{0}=\right.$ $0), g\left(\alpha_{0}\right)=\pi \alpha_{0} c_{n}^{2} F^{2}\left(\alpha_{0}\right)=0$, e $g^{\prime}\left(\alpha_{0}\right)=\pi c_{n}^{2} F^{2}\left(\alpha_{0}\right)$. As cargas de ruptura são gravadas e os resultados são apresentados na Tabela A1 do Apêndice e graficamente na Figura 3. Com base nos coeficientes de regressão, $A$ e $C$, pode-se determinar os parâmetros $K_{\text {lf }}$ e $\mathcal{C}_{f}$ de acordo com a equação (3). Os resultados são apresentados na Tabela 1 juntamente com os coeficientes de variação $(\mathrm{CV}=$ média/desvio padrão). Estes podem ser aproximados por $\mathrm{CV}\left[K_{l f}\right]=0,5 \times \mathrm{CV}[A] \mathrm{e}$ $\mathrm{CV}\left[c_{f}\right]=\left[(\mathrm{CV}[A])^{2}+(\mathrm{CV}[\mathrm{C}])^{2}\right]^{1 / 2}$ (Bazant e Kazemi, 1990b). Uma maior variação é encontrada em $c_{f}$ que é mais sensível aos erros experimentais e numéricos do que $K_{\text {If }}$. Conforme indicado na equação (3), enquanto $c_{f}$ depende de ambos $g\left(\alpha_{0}\right)$ e $g^{\prime}\left(\alpha_{0}\right), K_{I f}$ depende apenas de $g\left(\alpha_{0}\right)$. Também, o ponto de interseção de uma linha de regressão com o eixo y é mais sensível a dispersão de dados do que o seu coeficiente angular.

Tabela 1: Parâmetros de fratura

\begin{tabular}{cccc}
\hline $\begin{array}{c}K_{I f} \\
\left(\mathrm{MPa} \cdot \mathrm{m}^{1 / 2}\right)\end{array}$ & $\begin{array}{c}\mathrm{CV}\left[K_{I f}\right] \\
\%\end{array}$ & $\begin{array}{c}c_{f} \\
(\mathrm{~mm})\end{array}$ & $\begin{array}{c}\mathrm{CV}\left[\mathcal{c}_{f}\right] \\
\%\end{array}$ \\
\hline 1,195 & 3,9 & 40,7 & 13,8 \\
\hline
\end{tabular}

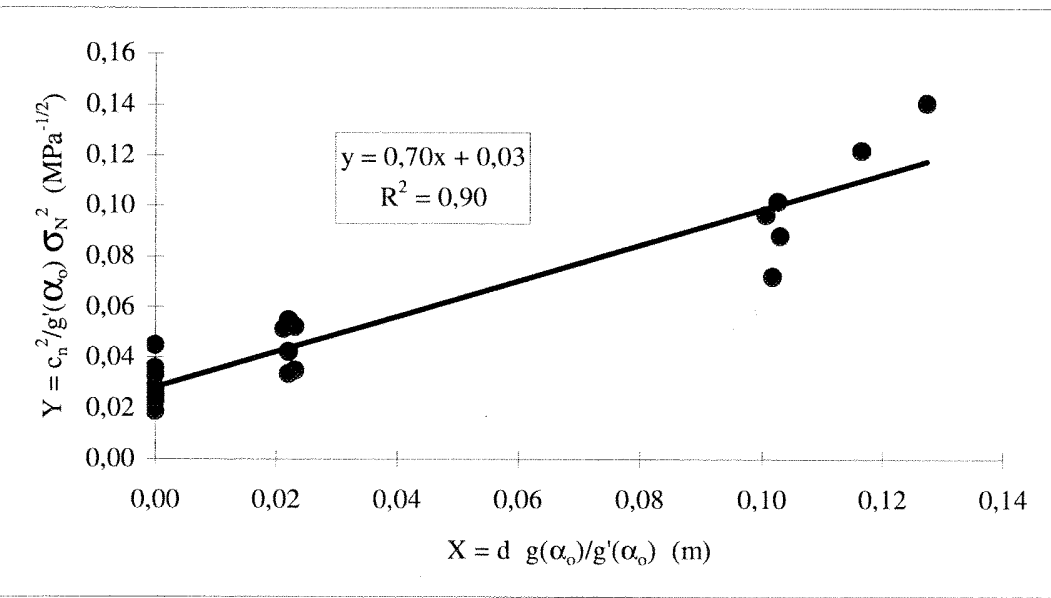

Figura 3: Resultados dos ensaios 
O método discutido foi aplicado com outras bateladas de concreto. Os valores dos parâmetros de fratura e da resistência à tração calculada são consistentes com os dados experimentais reportados na literatura internacional. Os resultados apresentam $R^{2}$ acima de 0,80 . Estes valores diminuem bastante quando utiliza-se o método na determinação dos parâmetros de fratura para concretos de pouca idade (até 12 horas). Isto é atribuído ao fato de que nestes concretos os caminhos de propagação da trinca são menos consistentes. Vale notar que não se observa fratura do agregado quando se rompe o corpo de prova nas primeiras horas. Ao contrário, aos 28 dias, a ligação argamassa-agregado é mais forte e é observado fratura do agregado no caminho da trinca. De qualquer forma, é interessante observar que os valores dos parâmetros de fratura são inferiores para concretos de pouca idade, indicando que estes são mais frágeis, ou seja, mais propensos a propagação de trincas.

\section{APLICAÇÕES}

\subsection{Determinação da Resistência do Concreto}

A resistência à tração de qualquer peça é determinada pela equação (3) desde que se conheça $K_{l f}$ e $c_{f}$, que caracterizam o material, e $g(\alpha)$, que caracteriza a geometria da peça. Para geometrias simples, como a do cilindro, existem funções disponíveis na literatura. A seguir, a resistência à tração de um corpo de prova cilíndrico é determinada a partir dos parâmetros de fratura da Tabela 1 como exemplo ilustrativo. Para a determinação da resistência à tração do mesmo material (mesmos $K_{\text {If }}$ e $c_{f}$ ) em outras geometrias é necessário que se determine a função de geometria correspondente. Para o cilindro em questão, os seguintes dados são necessários para a utilização da lei do efeito de tamanho:

(1) $c_{n}=2 / \pi$ uma vez que $\sigma_{N}=2 P /[\pi b d]$;

(2) $g(\alpha)$ e sua derivada são obtidas da relação $g(\alpha)=\pi \alpha c_{n}^{2} F^{2}(\alpha)$, onde $F(\alpha)$ é dado pela equação (5);

(3) O valor da razão dada pelo comprimento inicial da trinca/dimensão característica da peça, ou seja, $\alpha_{o}=a_{o} / d$;

(4) Parâmetros de fratura, $K_{I f}$ e $c_{f}$ (Tabela 1). 
Com a entrada de dados acima, e variando $\alpha_{0}$ de 0 a 0,9 na equação (3), obtêm-se a curva apresentada na Figura 4. Note que a resistência decresce dramaticamente a medida que a razão $\alpha_{0}$ se aproxima de 1 . É interessante notar que o valor de $\sigma_{N}\left(\alpha_{0}=0\right)$ determinado a partir dos parâmetros de fratura é consistente com o valor da resistência à tração indireta obtida no corpo de prova sem trinca inicial (ilustrado na Figura 4 por uma quadrado). Bazant e Li (1996) sugerem, com base em investigações em corpos de prova prismáticos, a substituição de $c_{f}$ na equação (3), por $c_{f}^{*}=1,4 c_{f}$ no caso da ausência da trinca inicial no corpo de prova. Este ajuste no tamanho crítico da zona de processamento da fratura diminui o valor da resistência quando $\alpha_{0}=$ 0. A Figura 4 bem como os demais resultados obtidos em Soares (1997) indicam que tal fator não é necessariamente válido para outras geometrias de corpos de prova, uma vez que para os cilindros investigados, a utilização de $c_{f}^{*}=c_{f}$ reproduziu fielmente a resistência à tração indireta nesta geometria.

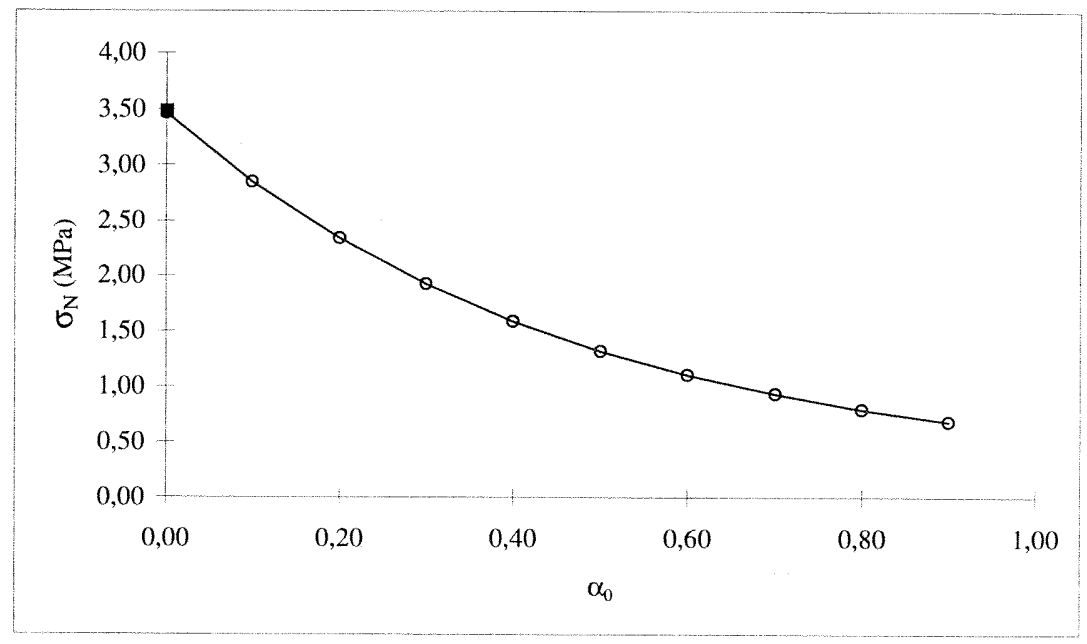

Figura 4: Resistência à tração em corpos de prova cilíndricos

Para obter um gráfico $\log \left(\sigma_{N}\right) \times \log (d)$ conforme mostrado na Figura 1, a abordagem apresentada por Bazant e Pfeiffer (1987) pode ser usada. Um valor específico de $\alpha_{0}$ é escolhido e a dimensão $d$ da peça é variada de um valor próximo de zero até uma valor que torne o número de fragilidade $\beta \approx 10$. A resistência à tração para $\alpha_{0}=0$, que 
corresponde a resistência à tração indireta, é designada por $B f_{u}$ para ser consistente com a terminologia usada por Bazant e Pfeiffer. O gráfico $\log \left(\sigma_{N} / B f_{u}\right) \times \log \left(d / d_{a}\right)$, onde $d_{a}$ é o tamanho máximo do agregado (25,4 mm no caso), é mostrado na Figura 5 . Note que dois valores de $\alpha_{0}(0,2$ e 0,6$)$ são usados para indicar a diferença na resistência nominal quando se considera diferentes comprimentos iniciais da trinca. Maiores valores de $\alpha_{0}$ correspondem a menores valores de resistência. Nota-se também que a resistência nominal $\sigma_{N}$ decresce com o aumento do tamanho $d$ da peça. A Figura 5 inclui ainda a reta inclinada correspondente a MFLE. O coeficiente angular desta reta é $-1 / 2$ e o ponto de interseção com o eixo y é obtido avaliando $\log \left(\sigma_{N} / B f_{u}\right)$ e $\log \left(d / d_{a}\right)$ para valores elevados de $d$ (correspondente a um número de fragilidade $\beta \geq 10$ ) e obtendo $C$ de $\log \left(\sigma_{N} / B f_{u}\right)=-1 / 2 \times \log \left(d / d_{a}\right)+C$.

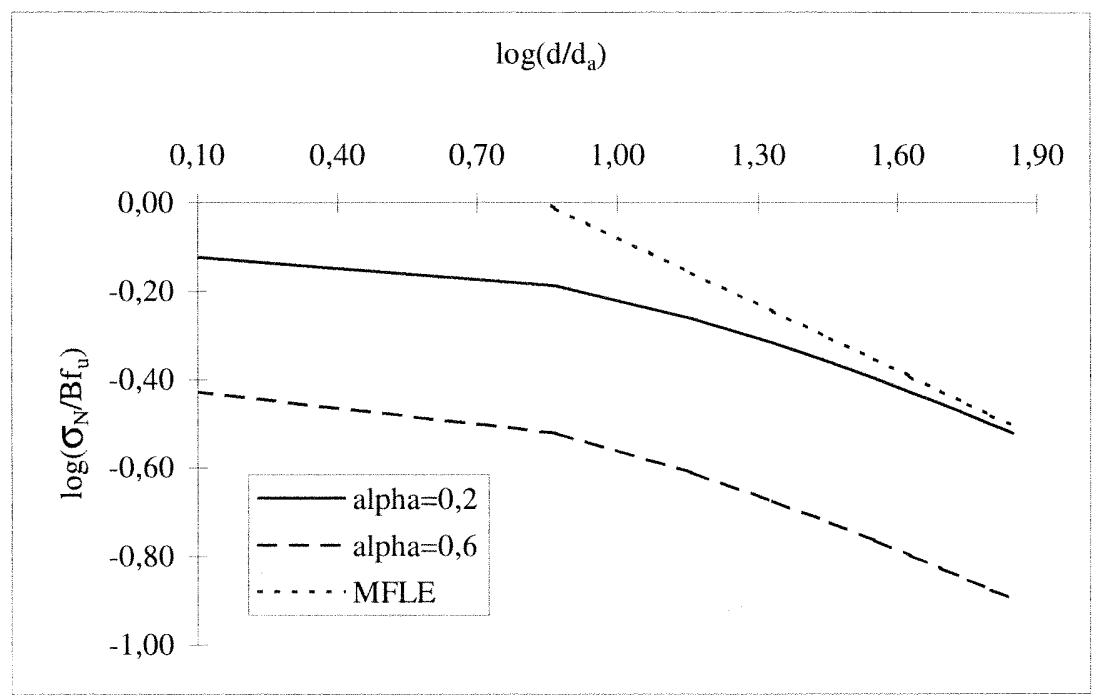

Figura 5: Efeito do tamanho em corpos de prova cilíndricos de concreto 


\subsection{Determinação da Profundidade de Corte em Placas de Concreto}

Uma aplicação mais direta da mecânica da fratura não linear a pavimentos de concreto se dá na determinação da profundidade de corte de placas. Tratando-se de requerimentos de corte em placas de concreto, preocupa-se principalmente com tensões desenvolvidas nas primeiras horas do concreto. Antes do carregamento devido ao tráfego, as tensões são principalmente devidas as mudanças de temperatura e umidade no concreto. Estas tensões podem ser analisadas conjuntamente considerando-se as deformações correspondentes causadas por um gradiente de temperatura equivalente entre o topo e o fundo da placa (Soares, 1997). Apesar da distribuição de temperatura em placas de concreto ser tipicamente não linear, para manter a simplicidade da análise, neste artigo é considerada uma distribuição linear de temperatura. A análise das tensões induzidas por um gradiente linear de temperatura em placas restringidas foi tratada por Westergaard (1927) e Bradburry (1938). Rufino (1997) é uma referência nacional que apresenta uma discussão sobre as tensões térmicas em placas de concreto. Em uma placa longa e larga repousando numa fundação Winkler, a tensão máxima de empenamento $\left(\sigma_{o}\right)$ distante da borda é a mesma em todas as direções horizontais:

$$
\sigma_{0}=\frac{E \alpha_{t} d T}{2(1-v)}
$$

onde: $E$ e v são o módulo de elasticidade e o coeficiente de Poisson, respectivamente, $\alpha_{t}$ é o coeficiente de dilatação térmica do concreto, e $d T$ é a diferença de temperatura entre o topo e o fundo da placa. $E$ é tipicamente obtido através de correlação com a resistência a compressão do concreto, $v$ é freqüentemente tomado como 0,15 , e $\alpha_{t}$ depende do tipo de agregado do concreto. Um valor típico quando se usa brita como agregado é $6,0 \times 10^{-6} /{ }^{\circ} \mathrm{C}$ (Mindess e Young, 1981). Gradientes de temperatura $(d T / h)$ entre 0,055 a $0,077^{\circ} \mathrm{C}$ por $\mathrm{mm}$ de profundidade da placa são encontrados (Rufino, 1997). A tensão máxima, função de $E$, e portanto do tempo, pode ser então comparada com a resistência, também função do tempo, obtida a 
partir dos ensaios de tração indireta em corpos de prova de laboratório.

Para se considerar o efeito da espessura da placa na especificação da profundidade de corte, a abordagem tratada em Soares e Zollinger (1997a) compara valores típicos de tensões térmicas em pavimentos à resistência do concreto (considerando a geometria e o tamanho do pavimento) conforme esta última varia com a razão profundidade de corte/espessura da placa $(\alpha)$. Na aplicação apresentada a seguir é considerado um concreto de 12 horas, $\operatorname{com} K_{I f}=0,144 \mathrm{MPa} \cdot \mathrm{m}^{1 / 2}$ e $c_{f}=$ $9,4 \mathrm{~mm}$. Uma extrapolação linear foi feita considerando-se um concreto de 6 horas com $K_{I f}=0,072 \mathrm{MPa} \cdot \mathrm{m}^{1 / 2}$ e $c_{f}=4,7 \mathrm{~mm}$, para ilustrar o efeito do tempo na especificação da profundidade de corte.

A resistência $\sigma_{N}$ em função de $\alpha$, conforme configurada numa placa de concreto serrada, pode ser obtida da equação (2). O fator de geometria da placa, fornecido em Zollinger et al. (1993) é $F(\alpha)=1,12$ $+0,203 \alpha-1,197 \alpha^{2}+1,930 \alpha^{3}$. O resultado é ilustrado na Figura 6 para placas de concretos de duas idades diferentes (6 e 12 horas) e duas espessuras $h$ diferentes $(15,24 \mathrm{~cm}$ e $45,72 \mathrm{~cm})$. As quatro linhas horizontais na Figura correspondem as tensões máximas para diferentes condições de gradiente de temperatura e tempo conforme dados da Tabela 2 com $\alpha_{t}$ considerado $6,0 \times 10^{-6} /{ }^{\circ} \mathrm{C}$.

Tabela 2: Tensões máximas numa placa de concreto

\begin{tabular}{lcccc}
\hline$D t\left({ }^{\circ} \mathrm{C}\right)$ & 5,56 & 5,56 & 11,11 & 11,11 \\
Tempo (horas) & 6 & 12 & 6 & 12 \\
$E(\mathrm{MPa})$ & 7.590 & 10.340 & 7590 & 10.340 \\
$\sigma_{\max }(\mathrm{MPa})$ & 0,15 & 0,20 & 0,30 & 0,40 \\
\hline
\end{tabular}




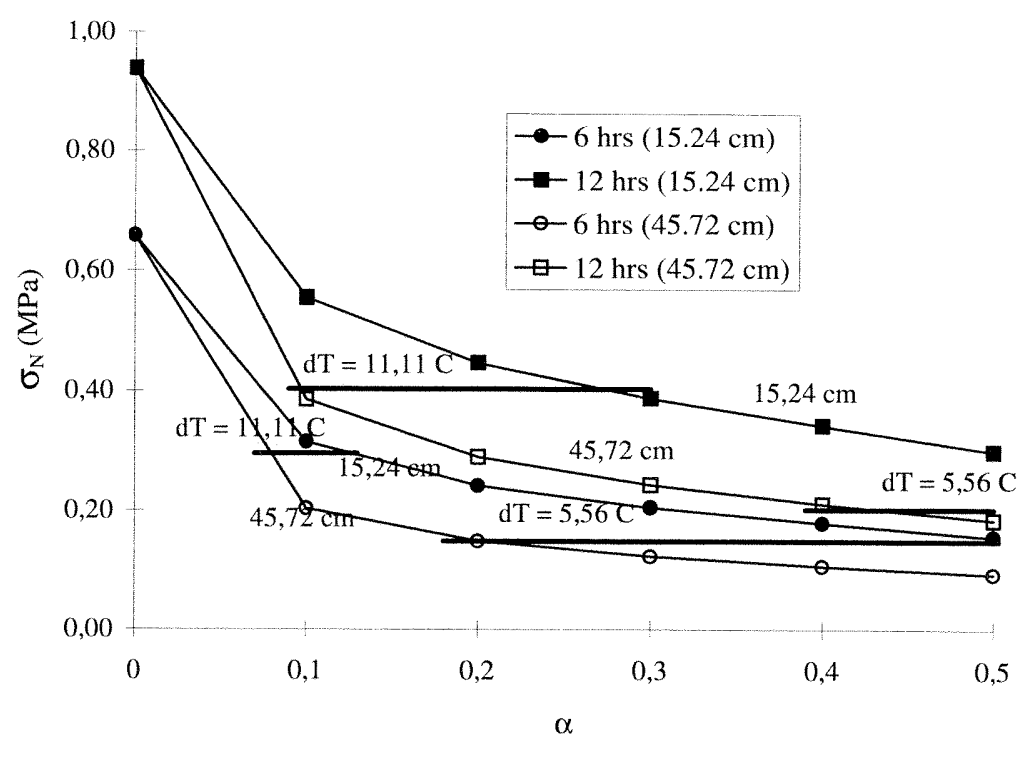

Figura 6: Profundidade de corte e requerimento de tempo considerando a tensão máxima

Para se determinar a profundidade de corte a partir da Figura 6, precisa-se determinar o ponto onde a linha horizontal de máxima tensão térmica intercepta a curva de resistência em questão. A Tabela 3 mostra a profundidade de corte requerida para os níveis de tensão considerados na Tabela 2 . Uma vez que a placa mais delgada possui maior resistência à tração para uma determinada razão $\alpha$, devido ao efeito de tamanho, ela sempre requer uma profundidade de corte proporcionalmente maior do que a placa mais espessa quando se considera um mesmo nível de tensão. Devido aos maiores valores dos parâmetros de fratura, uma placa de 12 horas exige um $\alpha$ maior que uma placa de 6 horas. Nota-se ainda que níveis de tensão mais baixos acentuam o efeito da espessura da placa e da idade do concreto na razão $\alpha$. É muito importante observar que quando um nível de tensão baixo é encontrado, como no caso de 0,15 MPa e 0,20 $\mathrm{MPa}$, razões $\alpha$ maiores que $h / 3$ podem ser necessárias para que a trinca se propague a partir da ponta do corte através de toda a profundidade da placa a fim de evitar trincamento aleatório. Maiores 
discussões sobre o tempo e a profundidade de corte são encontradas em Soares (1997) e Soares e Zollinger (1997a).

Tabela 3: Profundidade de corte requerida

\begin{tabular}{lcccccccc}
\hline$\sigma_{\max }(\mathrm{MPa})$ & 0,15 & & 0,30 & & 0,20 & \multicolumn{3}{c}{0,40} \\
Tempo (horas) & 6 & & 6 & & 12 & & 12 & \\
Espessura da & 15,24 & 45,72 & 15,24 & 45,72 & 15,24 & 45,72 & 15,24 & 45,72 \\
placa, $h(\mathrm{~cm})$ & & & & & & & \\
$\alpha$ requerido & 0,50 & 0,20 & 0,12 & 0,08 & $>0,50$ & 0,43 & 0,27 & 0,10 \\
$\begin{array}{l}\text { Profundidade } \\
\text { de Corte }(\mathrm{cm})\end{array}$ & 7,7 & 9,2 & 1,9 & 3,7 & $>7,7$ & 19,7 & 4,2 & 4,6 \\
\hline
\end{tabular}

\section{CONCLUSÕES}

Este artigo apresenta um ensaio de laboratório simplificado para se determinar os parâmetros de fratura do concreto a partir de corpos de prova cilíndricos modificados. É mostrado como a resistência do concreto pode ser determinada como uma função da dimensão da peça (por exemplo, espessura de uma placa) e também como função da razão $\alpha_{0}=$ comprimento da trinca inicial/dimensão característica. Esta é a grande vantagem do uso da teoria da mecânica da fratura sobre a teoria da ruptura uma vez que esta última não reconhece o efeito do tamanho e geometria da peça na resistência do material. A utilização dos parâmetros de fratura na determinação da profundidade de corte em pavimentos de concreto também foi apresentada. Foi mostrada a importância de se considerar a espessura da placa e o estado de tensões atuante nesta placa para a determinação da profundidade de corte. 


\section{APÊNDICE}

Tabela A1: Dados de entrada para a determinação dos parâmetros de fratura

\begin{tabular}{ccccccccc}
\hline $\begin{array}{c}\text { Corpo } \\
\text { de } \\
\text { Prova }\end{array}$ & $\begin{array}{c}b \\
(\mathrm{~mm})\end{array}$ & $\begin{array}{c}2 a_{o} \\
(\mathrm{~mm})\end{array}$ & $\begin{array}{c}P \\
(\mathrm{~N})\end{array}$ & $o$ & $g(o)$ & $g^{\prime}\left(o_{0}\right)$ & $\begin{array}{c}X=d\left(g / g^{\prime}\right) \\
(\mathrm{m})\end{array}$ & $\begin{array}{c}Y=c^{2} /\left(g^{\prime} \mathrm{N}^{2}\right) \\
\left(1 / \mathrm{MPa}^{2}\right)\end{array}$ \\
\hline \multirow{6}{*}{ Tipo 1 } & 94,3 & 0,0 & 69.745 & 0,0 & 0,0 & 1,183 & 0,0 & 0,0359 \\
& 98,8 & 0,0 & 75.794 & 0,0 & 0,0 & 1,183 & 0,0 & 0,0334 \\
& 102,5 & 0,0 & 86.825 & 0,0 & 0,0 & 1,183 & 0,0 & 0,0274 \\
& 102,5 & 0,0 & 85.980 & 0,0 & 0,0 & 1,183 & 0,0 & 0,0279 \\
& 97,8 & 0,0 & 90.517 & 0,0 & 0,0 & 1,183 & 0,0 & 0,0229 \\
& 96,3 & 0,0 & 98.078 & 0,0 & 0,0 & 1,183 & 0,0 & 0,0189 \\
& 94,3 & 0,0 & 62.272 & 0,0 & 0,0 & 1,183 & 0,0 & 0,0450 \\
& 98,3 & 0,0 & 89.138 & 0,0 & 0,0 & 1,183 & 0,0 & 0,0239 \\
& 102,5 & 0,0 & 89.983 & 0,0 & 0,0 & 1,183 & 0,0 & 0,0255 \\
\hline \multirow{6}{*}{ Tipo 2 } & 95,0 & 24,2 & 53.376 & 0,159 & 0,201 & 1,434 & 0,0213 & 0,0513 \\
& 101,8 & 27,0 & 55.511 & 0,177 & 0,228 & 1,499 & 0,0232 & 0,0521 \\
& 99,0 & 27,1 & 65.964 & 0,178 & 0,228 & 1,500 & 0,0232 & 0,0349 \\
& 85,5 & 25,4 & 46.037 & 0,167 & 0,212 & 1,460 & 0,0221 & 0,0549 \\
& 83,3 & 25,4 & 51.063 & 0,167 & 0,212 & 1,460 & 0,0221 & 0,0423 \\
& 90,0 & 25,4 & 62.094 & 0,167 & 0,212 & 1,460 & 0,0221 & 0,0334 \\
\hline \multirow{6}{*}{ Tipo 3 } & 97,0 & 38,1 & 47.816 & 0,250 & 0,657 & 0,994 & 0,1007 & 0,0962 \\
& 99,0 & 37,7 & 48.038 & 0,248 & 0,654 & 0,971 & 0,1027 & 0,1015 \\
& 102,3 & 37,7 & 53.465 & 0,247 & 0,654 & 0,968 & 0,1030 & 0,0877 \\
& 94,0 & 33,9 & 43.946 & 0,222 & 0,633 & 0,757 & 0,1273 & 0,1403 \\
& 98,5 & 35,6 & 46.971 & 0,233 & 0,641 & 0,840 & 0,1165 & 0,1217 \\
& 104,4 & 37,9 & 59.870 & 0,248 & 0,655 & 0,981 & 0,1018 & 0,0719 \\
\hline
\end{tabular}




\section{REFERÊNCIAS BIBLIOGRÁFICAS}

ABNT (1994) NBR 7222 - Argamassa e concreto - determinação da resistência à tração por compressão diametral de corpos de prova cilíndricos. NBR 7222, Associação Brasileira de Normas Técnicas, Rio de Janeiro.

ASTM (1995) Standard Test Method for Splitting Tensile Strength of Cylindrical Concrete Specimens. C496-90, Philadelphia, PA.

Bazant, Z.P. (1984) Size Effect in Blunt Fracture: Concrete, Rock, and Metal. Journal of Engineering Mechanics, ASCE, 110(4), 518-535. Bazant, Z.P. e Oh, B.H. (1983) Crack Band Theory for Fracture of Concrete. Materials and Structures, 16, 155-177.

Bazant, Z.P. e Pfeiffer, P.A. (1987) Determination of Fracture Energy from Size Effect and Brittleness Number. ACI Materials Journal, 84(6), 463-480.

Bazant, Z.P. e Kazemi, M.T. (1990a) Determination of Fracture Energy, Process Zone Length and Brittleness Number from Size Effect, with Application to Rock and Concrete. International Journal of Fracture, 44(2), 111-131.

Bazant, Z.P. e Kazemi, M.T. (1990b) Size Effect in Fracture of

Ceramics and its Use to Determine Fracture Energy and Effective Process Zone Length. Journal of American Ceramic Society, 73(7), 1841-1853.

Bazant, Z.P. e Li, Z. (1996) Zero-brittleness Size Effect Method for

One-size Fracture Test Concrete. Journal of Engineering Mechanics, ASCE, 122(5), 458-468.

Bradbury, R.D. (1938) Reinforced Concrete Pavements. Wire Reinforcement Institute, Washington, DC.

Broek, D. (1986) Elementary Engineering Fracture Mechanics. Kluwer Academic Publishers, Dordrecht, The Netherlands.

Carneiro, F.L.L.B. (1953) Concrete Tensile Strength. RILEM Bulletin, 13, 103-107.

Chan, S.K., Tuba, I.S. e Wilson, W.K. (1970) On the Finite Element Method in Linear Fracture Mechanics. Engineering Fracture Mechanics, 2, 1-17.

Elices, M. e Planas, J. (1989) Material models. Fracture Mechanics of Concrete Structures, L. Elfgren, ed., Chapman and Hall, London, UK, 16-66. 
Hillerborg, A., Modeer, M. e Peterson, P.E. (1976) Analysis of Crack Formation and Crack Growth in Concrete by Means of Fracture Mechanics and Finite Elements. Cement and Concrete Research, 6(6), 773-782.

Huang, Y.H. (1974) Finite Element Analysis of Slabs on Elastic Solids. Transportation Engineering Journal, ASCE, 100(TE2), 403-416.

Ioannides, A.M., Thompson, M.R. e Barenberg, E.J. (1985) The Westergaard Solutions Reconsidered. Presented at the January 1985 Annual Meeting of the Transportation Research Board, Washington, DC.

Ioannides, A.M. (1997) Fracture Mechanics in Pavement Engineering, the Specimen Size-effect. Transportation Research Record 1568, 10-16.

Jenq, Y. e Shah, S.P. (1985) Two Parameter Fracture Model for Concrete. Journal of Engineering Mechanics, 111(10), 1227-1241.

Lin, S.T. e Folias, E.S. (1975) On the Fracture of Highway Pavements. International Journal of Fracture, 11(1), 93-106.

Mindess, S. e Young, J.F. (1981) Concrete. Prentice-Hall, Inc., Englewood Cliffs, NJ.

Nallathambi, P. e Karihaloo, B.L. (1986) Determination of Specimensize Independent Fracture Toughness of Plain Concrete. Magazine of Concrete Research, 38(135), 67-76.

Ojdrovic, R.P. e Petroski, H.J. (1987) Fracture Behavior of Notched Concrete Cylinder. Journal of Engineering Mechanics, ASCE, 113(10), 1551-1563.

Parks, D.M. (1974) A Stiffness Derivative Finite Element Technique for Determination of Crack Tip Stress Intensity Factors. International Journal of Fracture, 10(4), 487-501.

Pickett, G. e Ray, G.K. (1951) Influence Chart for Concrete Pavement. Transactions, ASCE, 116, 49-73.

Pinto, S. (1991) Estudo do comportamento à fadiga de misturas betuminosas e aplicação na avaliação estrutural de pavimentos, Tese de Doutorado, COPPE/UFRJ, Rio de Janeiro.

Planas, J. e Elices, M. (1989) Size-effect in Concrete Structures: Mathematical Approximations and Experimental Validation. Cracking and damage: strain localization and size effect, J. Mazars and Z.P. Bazant, eds., Elsevier, London, UK, 462-476. 
Planas, J. e Elices, M. (1990) Fracture Criteria for Concrete: Mathematical Approximations and Experimental Validation. Engineering Fracture Mechanics, 35(1), 87-94.

Ramsamooj, D.V. (1993) Fracture of Highway and Airport Ppavements. Engineering Fracture Mechanics, 44(4), 609-626.

RILEM (1990) Size-effect Method for Determining Fracture Energy and Process Zone Size of Concrete. Materials and Structures, 23(138), 461-465.

Rodrigues, R. (1991) Estudo do Trincamento dos Pavimentos. Tese de Doutorado, COPPE/UFRJ, Rio de Janeiro.

Rufino, D.M. (1997) Estudo dos Procedimentos de Dimensionamento e dos Novos Programas de Análise de Tensões em Pavimentos de Concreto. Dissertação de Mestrado, COPPE/UFRJ, Rio de Janeiro.

Shah, S.P., Swartz, S.E. e Ouyang, C. (1995) Fracture Mechanics of Concrete. John Wiley \& Sons, Inc., New York, NY.

Soares, J.B. (1997) Concrete Characterization by Fracture Mechanics and Selected Pavement Aplications, Tese de Doutorado, Texas A\&M University, College Station, TX.

Soares, J.B. e Zollinger, D.G. (1997a) Strength Characterization and a Basis for Sawcutting Requirements for Jointed Concrete Pavements. 6a. International Purdue Conference on Concrete Pavement Design and Material for High Performance, Volume 2, 41-61.

Soares, J.B. e Zollinger, D.G. (1997b) A Compatible Approach to Concrete Strength Characterization in the Design and Construction of Concrete Pavements." Transportation Research Record 1568, 89-95.

Swartz, S.E. e Refai, T.M.E. (1988) Influence of Size Effects on Opening Mode Fracture Parameters for Precracked Concrete Beams in Bending. Fracture of concrete and rock, S.P. Shah and S.E. Swartz, eds., Springer-Verlag, New York, NY, 242-254.

Tada, H., Paris, P.C. e Irwin, G.R. (1985) The Stress Analysis of Cracks Handbook. 2nd Edition, Paris Productions Inc., St. Louis, MO.

Tang, T., Shah, S.P. e Ouyang, C. (1992) Fracture Mechanics and Size Effect of Concrete in Tension. Journal of Structural Engineering, ASCE, 118(11), 3169-3185. 
Tang, T., Bazant, Z.P., Yang, S. e Zollinger, D.G. (1996) Variablenotch One-size Test Method for Fracture Energy and Process Zone Length. Engineering Fracture Mechanics, 55(3), 383-404.

Westergaard, H.M. (1926) Stresses in Concrete Pavements Computed by Theoretical Analysis. Public Roads, 7, 25-35.

Westergaard, H.M. (1927) Analysis of Stresses in Concrete Roads Caused by Variations of Temperature. Public Roads, 8(3), 5460.

Wright, P.J.F. e Garwood, F. (1952) The Effect of Method of Test on the Flexural Strength of Concrete. Magazine of Concrete Research, 11, 67-76.

Zollinger, D.G., Tang, T. e Yoo, R.H. (1993) Fracture Toughness of Concrete at Early Ages. ACI Materials Journal, 90(5), 463-471.

Zollinger, D.G., Tang, T. e Dapeng, X. (1994) Sawcut Depth Considerations for Jointed Pavement Based on Fracture Mechanics Analysis. Transportation Research Record 1449, 91100 .

\section{Endereço dos autores:}

\section{Jorge Barbosa Soares}

Departamento de Engenharia de Transportes

Universidade Federal do Ceará

Campus do Pici - Bloco 703 - Fortaleza, CE 60.455-760

E-mail: jsoares@det.ufc.br 